\title{
A Surgical Perspective on Ischemic Mitral Regurgitation: Tethering or Prolapse? Going Back to Papillary Muscles Anatomy. What the Surgeons Really Need to Know
}

\author{
Francesco Nappi ${ }^{*}$, Cristiano Spadaccio ${ }^{2}$, Christophe Acar ${ }^{3}$ \\ ${ }^{1}$ Cardiac Surgery Centre Cardiologique du Nord de Saint-Denis, Paris, France \\ ${ }^{2}$ Department of Cardiothoracic Surgery, Golden Jubilee National Hospital, \\ Glasgow, UK \\ ${ }^{3}$ Department of Cardiovascular Surgery, Hôpital de la Salpétrière, Paris, France \\ Email: ${ }^{*}$ francesconappi2@gmail.com
}

Received 13 May 2015; accepted 14 July 2015; published 17 July 2015

Copyright (C 2015 by authors and Scientific Research Publishing Inc.

This work is licensed under the Creative Commons Attribution International License (CC BY). http://creativecommons.org/licenses/by/4.0/

(c) (i) Open Access

\section{Abstract}

More than one third of patients with ischemic mitral regurgitation (IMR) present a valve prolapse whose mechanism is subtended by a papillary injury. The recent literature is pointing at a regional ventricular injury or wall motion abnormality rather than a global $\mathrm{LV}$ dysfunction as responsible for IMR and the presence of localized valve prolapse related to papillary dysfunction is additionally supporting this idea. Leaflet tethering or prolapse in these patients is subtended by lesion of the papillary muscle (PM) per se rather than its dysfunction secondary to regional and global ventricle enlargement. Identification of this type of lesion is difficult and can be overlooked. Morphological characteristics and anatomical variability of the papillary muscles determine their different susceptibility ischemic damage and dysfunction. Pioneering work in mitral anatomy shows a range of morphological diversity of PM anatomy and leads to an anatomical classification with important implications in IMR surgery. New methods of investigation, as multidetector computed tomography or magnetic resonance provide a very accurate and proper identification of the morphological pattern of the subvalvular apparatus, which is crucial for a long-lasting and successful surgical correction. The involvement of PM in the pathophysiology of IMR not only in terms of their functional anomaly, but also of their effective anatomical aspects and characteristics is increasingly emerging. The modern advancements of imaging techniques can guide the preoperative surgical planning and the surgeon needs to be aware of morphological features of the subvalvular apparatus and combine these findings with echographic functional parameters before embarking in complex mitral repairs.

"Corresponding author.

How to cite this paper: Nappi, F., Spadaccio, C. and Acar, C. (2015) A Surgical Perspective on Ischemic Mitral Regurgitation: Tethering or Prolapse? Going Back to Papillary Muscles Anatomy. What the Surgeons Really Need to Know. World Journal of Cardiovascular Surgery, 5, 63-69. http://dx.doi.org/10.4236/wjcs.2015.57011 
Keywords

Ischemic Mitral Regurgitation, Papillary Muscle, Valve Prolapse, Imaging Techniques

\section{Introduction}

Current debate on ischemic mitral regurgitation (IMR) is animated by several studies introducing parameters or variables predicting prognosis or outcome of mitral repair. Kron and colleagues reported a set of clinical and echocardiographic variables able to predict recurrence of MR after surgical repair [1]. However, general results of valve repair in IMR remain frustrating dropping a veil of uncertainty on the actual benefit of this type of surgery [2]-[4]. Success of mitral repair relies on the comprehension of both the geometric modifications of the annulus and the morphology and physiopathology of papillary muscle (PM) [5].

\section{The Clinical Problem}

Since 2001, our group focused on the role of the PM in the pathophysiology of IMR and on the possibility to correct it through a direct action on the subvalvular apparatus. Restrictive mitral annuloplasty and papillary muscle approximation (PMA) was systematically performed in a series of patients undergoing surgery for IMR [6]. Later, other experiences have been reported about the procedures of papillary muscle relocation [7] [8] and papillary muscle imbrication [9]. Only many years later, Kalra et al. described the potential role of interpapillary muscle distance (IPMD) shortening in IMR enlightening new avenues in both the comprehension of the mechanisms underlying its pathogenesis and the potential for its surgical correction [10]. Their findings are supporting the idea, elegantly pointed out by Sanz et al., of a localized LV injury at the level of PM rather than a global LV dilation as pathogenic basis of IMR [11]. This concept was already perceived by Jouan et al. in 2004 when a $36 \%$ incidence of mitral prolapse with precise regional characterization was described in a large series of patients undergoing surgery for IMR. In this report, a close examination of the regional differences in the anatomical characteristics of the mitral prolapse during IMR together with a classification of the types of PM injury subtending the site-specific prolapse pathogenesis was reported [12]. Clearly, these descriptive findings were pointing at a regional ventricular injury or wall motion abnormality rather than a global LV dysfunction as responsible for IMR and the presence of localized valve prolapse is additionally supporting this idea. Today, according to American College of Cardiology guidelines for valvular heart disease [13], it is increasingly accepted that the post-ischemic adverse phenomenon of left ventricular remodelling promotes an imbalance between tethering forces and closing forces. The tethering forces are represented by annular dilatation, enlargement of the left ventricular chamber, abnormal displacement of the papillary muscles with apical and lateral migration. The closing forces are represented by reduction of LV contractility, global LV dyssyncrony, papillary muscle dyssyncrony and altered mitral systolic annular contraction [14] [15].

\section{Morphology and Pathophysiology}

In our experience in IMR even if valve motion restriction with annular dilatation was the most frequent functional abnormality, presence of primary lesion or dysfunction of PM leading to prolapse was surprisingly high (86.4\% of IMR patients found to have valve prolapse). Antero-lateral PM (ALPM) was involved in $18.2 \%$ and postero-medial PM (PMPM) in 63.8\% respectively. Figure 1 shows distribution of prolapse within the mitral valve. In $13.6 \%$ the prolapse was determined by the necrosis of a restricted area of the myocardium adjacent to the PM, which was responsible for its abnormal traction and of its dyssyncrony. In addition, all patients had some degree of annulus dilatation (functional type I) as well as systolic restricted leaflet motion on the remaining leaflets (type IIIb). Leaflet tethering or prolapse in these patients is subtended by the lesion of the PM per se rather than its dysfunction secondary to regional and global ventricle enlargement. Identification of this type of lesion is difficult and can be overlooked. Indeed, mechanisms at the root of this finding rely on the morphological characteristics and anatomical variability of the PM itself, which determines a different susceptibility to ischemic damage. Pioneering work in mitral anatomy showed a range of morphological diversity of PM anatomy and led to an anatomical classification with important implications in IMR surgery. The uneven coronary 
A2

A1

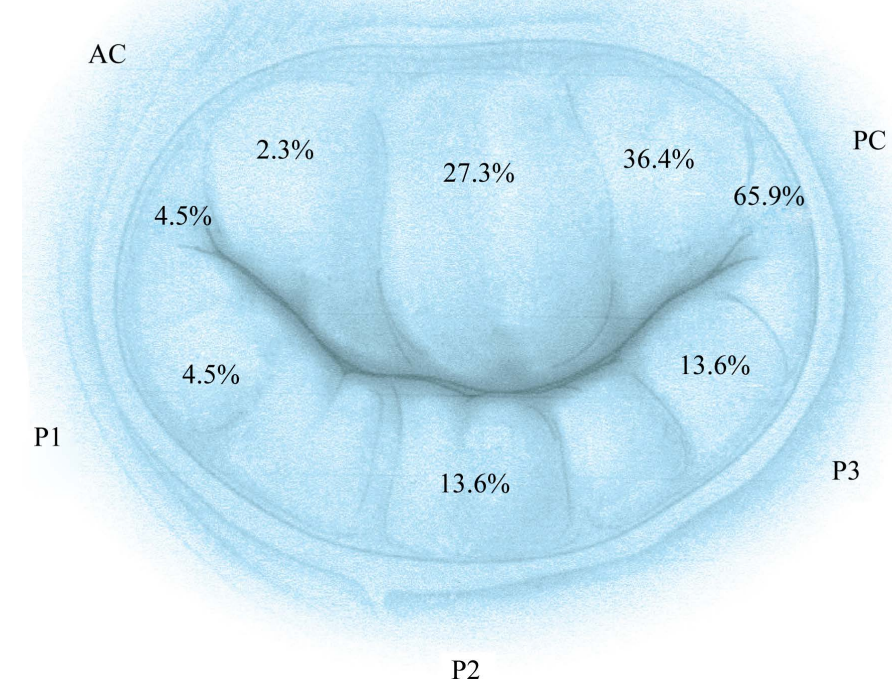

Figure 1. Distribution and incidence of prolapse within the mitral valve. The posteromedial commissure was involved in the majority of cases. The A3 portion of the anterior leaflet was prolapsed in onethird of the cases.

distribution and the difference of PM anatomy account for a heterogeneous morphological variability of the papillary damage. Asymmetric distribution of blood supply accounts for the rare involvement of the anterior PM, which is perfused by both the left anterior descending coronary artery and diagonal branch. In addition, the tension exerted by the chordae on this PM is relatively low due to its superficial location with regard to the annulus. Conversely, the posterior PM is more sensitive to ischemia (91\% of the cases in our series) because its blood supply relies on distal branches exclusively furnished by either the right coronary or the circumflex artery; furthermore, its location deep in the left ventricle subjects this muscle to a higher shear force. PM microcirculation relies in both an independent blood supply, provided by a well-identified arterial trunk perforating the PM from base to apex (Kugel's artery), and a segmental distribution [16]. However, features of microcirculation imbricate with anatomical characteristics of PM and the relative importance of one of the two circulatory systems depends on the morphology and position of the PM within the ventricle and on the presence muscular bridging, which favors collateralization. A classification of the various stages of segmentation is described in Figure 2. Clearly, the importance of the truncal system increases as the PM is more individualized from the ventricular wall, as in type IV-V (Figure 1) with the apex becoming more prone to rupture due to the fragility of its truncal blood supply and to the degree of physical stress. Partial papillary muscle rupture or elongation limited to a single head are therefore more likely to occur than in the case of the presence of multiple muscular bridges that guarantee an adequate collateral compensation. Mechanism of prolapse is shown in Figure 3. The morphology of the posterior PM, which is the usual site of ischemic injury, is more complex than the anterior PM and its subdivision into several heads is very frequent. Ischemic mitral prolapse is frequently caused by a partial papillary muscle rupture or elongation limited to a single head. Alternatively prolapse can be favored by an incomplete detachment of a head due to a rupture of its main insertion occurred while it remained fixed to the ventricle via muscular bridges ('incomplete' papillary muscle rupture).

\section{Clinical Use and Area of Uncertainty}

These determinants of papillary morphology require rigorous consideration when evaluating early and specific approaches to the treatment of IMR. This might achieve additional significance considering the reappraisal of surgical techniques involving intervention on subvalvular apparatus and aimed to correct altered geometry of the LV in IMR, as PM approximation (PMA) [6] PM imbrication [17] or PM relocation [7]. These techniques might be applicable with good results in the medium and long term only in case of favorable anatomy of the posterior 


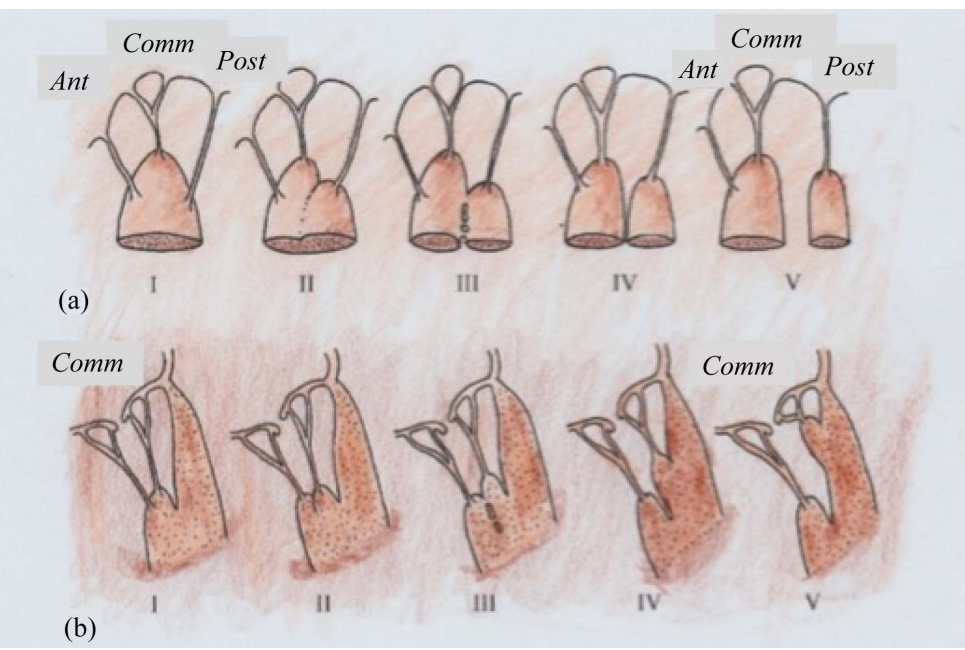

Figure 2. Segmentation and morphological types of papillary muscles. I, single uniform unit. II, groove with two apexes; III, fenestrations with muscular bridges; IV, complete separation in two adjacent heads; V, complete separation with two distant heads. Division can occur according to two directions: (a) Division in a sagittal plane leading to a separate posterior leaflet head. (b) Division in a coronal plane leading to a separate commissural head. Ant, anterior leaflet; Comm, commissure; Post, posterior leaflet.

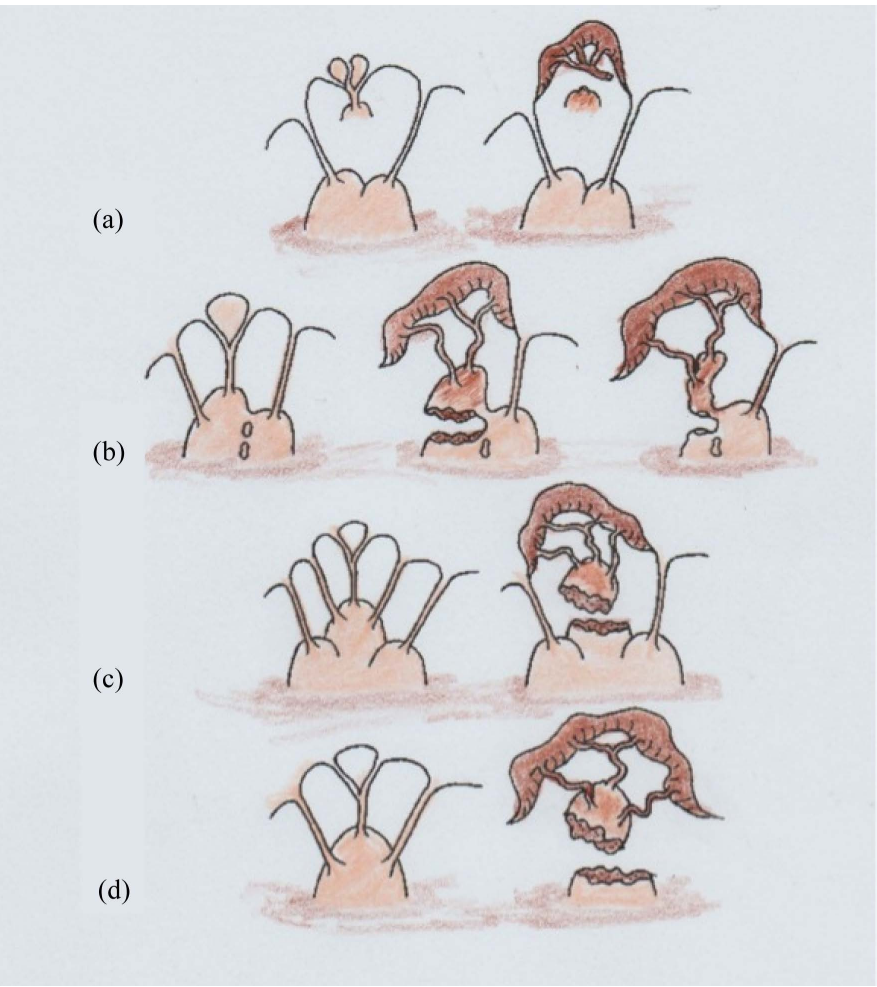

Figure 3. Mechanisms of ischemic mitral valve prolapse. (a) Necrosis of a separate commissural head (inserted close to the annulus) with rupture of the anchorage of the commissural chord. (b) Necrosis of a single head papillary muscle subdivided in multiple heads with partial rupture. (c) Necrosis of a fenestrated papillary muscle with detachment of its main insertion: "incomplete” rupture. With time, incomplete rupture mimics papillary muscle elongation. (d) Single papillary muscle with complete and total rupture. 


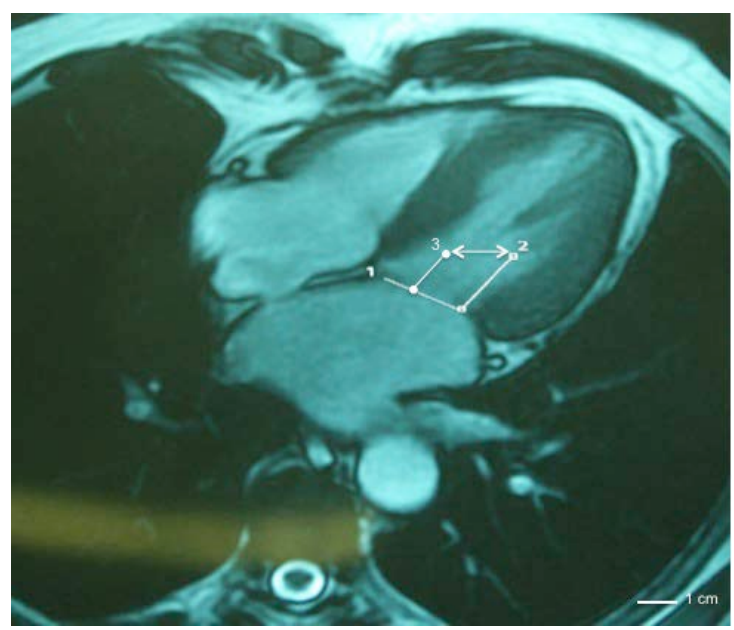

Figure 4. Cardiac MRI. 4 chambers view showing measurements of annulus and subvalvular apparatus. Asymmetric tethering of papillary muscle. 1) Annulus diameter. 2) Distance between postero-medial papillary muscle and annulus after post-remodeling papillary muscle displacement. 3) Distance between antero-lateral papillay muscle and annulus. The anterior papillary muscle is not involved in ischemic mitral injury and it is not displaced. Double arrow displays interpapillary muscle distance (IPMD).

medial papillary muscle, as is potentially found in type I-II -III morphology with well represented central body, while are less applicable for the remaining types IV-V (Figure 1). New methods of investigation, as multidetector computed tomography (MDCT) with 3D reconstruction or MRI provide a very accurate and proper identification of the morphological pattern of the subvalvular apparatus, which is crucial for a long-lasting and successful surgical correction [9] [18] (Figure 4). Additionally, unveiling the presence of a symmetric or an asymmetric tethering pattern of the mitral valve might further guide the choice of surgical strategy [19]. For instance, cases of global ventricular dilatation with increased sphericity due to the extensive MI would benefit from PM relocation but it is necessary to relocate both the muscles to the annulus. Conversely, in asymmetric tethering, the operation of PMA requires a careful evaluation of the extent of the approximation to be performed because the ALPM gives rise to the chordae directed to the anterior leaflet, which are responsible of the correct valve conformation and tenting angle, and its dislocation might result in excessive traction and restriction on this leaflet (seagull sign) [20]. On the other side, the PMPM originates the chordae to scallop P2 and P3 of the posterior leaflet being directly involved in posterior valve prolapse. Clearly, in both the situations a detailed preoperative imaging might be useful to elaborate the optimal surgical strategy and to plan the choice of the adequate subvalvular correction technique to be used.

\section{Recommendation}

In conclusion, the role of PM in the pathophysiology of IMR is increasingly emerging in the literature, but their involvement concerns not only their functional anomaly but also their effective anatomical aspects and characteristics. If some years ago assessment of the valvular and subvalvular status and the choice of the appropriate reparative strategy relied in the surgeon intraoperative analysis of the valve and tactile feedback [12], the modern advancements of imaging techniques allowed for an extremely detailed description of subvalvular anatomy and could guide the preoperative surgical planning. Even if these techniques are not readily available in every center and require specific radiological expertise, the surgeon needs to be aware of morphological features of the subvalvular apparatus and combine these findings with echographic functional parameters before embarking in complex mitral repairs.

\section{Source of Funding}

None. 


\section{Disclosures}

Authors declare no conflicts of interest.

\section{References}

[1] Kron, I.L., Hung, J., Overbey, J.R., Bouchard, D., Gelijns, A.C., Moskowitz, A.J., Voisine, P., O’Gara, P.T., Argenziano, M., Michler, R.E., Gillinov, M., Puskas, J.D., Gammie, J.S., Mack, M.J., Smith, P.K., Sai-Sudhakar, C., Gardner, T.J., Ailawadi, G., Zeng, X., O’Sullivan, K., Parides, M.K., Swayze, R., Thourani, V., Rose, E.A., Perrault, L.P., Acker, M.A. and Investigators, C. (2015) Predicting Recurrent Mitral Regurgitation after Mitral Valve Repair for Severe Ischemic Mitral Regurgitation. The Journal of Thoracic and Cardiovascular Surgery, 149, 752-761.

[2] Acker, M.A., Parides, M.K., Perrault, L.P., Moskowitz, A.J., Gelijns, A.C., Voisine, P., Smith, P.K., Hung, J.W., Blackstone, E.H., Puskas, J.D., Argenziano, M., Gammie, J.S., Mack, M., Ascheim, D.D., Bagiella, E., Moquete, E.G., Ferguson, T.B., Horvath, K.A., Geller, N.L., Miller, M.A., Woo, Y.J., D’Alessandro, D.A., Ailawadi, G., Dagenais, F., Gardner, T.J., O’Gara, P.T., Michler, R.E., Kron, I.L. for the CTSN (2014) Mitral-Valve Repair versus Replacement for Severe Ischemic Mitral Regurgitation. The New England Journal of Medicine, 370, 23-32. http://dx.doi.org/10.1056/NEJMoa1312808

[3] Nicolini, F., Agostinelli, A., Vezzani, A., Molardi, A., Benassi, F., Gallingani, A., Romano, G. and Gherli, T. (2015) Surgical Treatment for Functional Ischemic Mitral Regurgitation: Current Options and Future Trends. Acta Biomed, 86, 17-26.

[4] Jensen, H. (2015) Surgical Treatment of Functional Ischemic Mitral Regurgitation. Danish Medical Bulletin, 61, 3.

[5] Hajsadeghi, S., Samiee, N., Hosseini, S.S., Hassanzadeh, M. and Jafarian Kerman, S.R. (2015) Novel Echocardiographic Indices as Predictors of Immediate Recurrence after Undersized Ring Annuloplasty for Ischemic Mitral Regurgitation. Echocardiography. http://dx.doi.org/10.1111/echo.12879

[6] Rama, A., Nappi, F., Praschker, B.G. and Gandjbakhch, I. (2008) Papillary Muscle Approximation for Ischemic Mitral Valve Regurgitation. Journal of Cardiac Surgery, 23, 733-735. http://dx.doi.org/10.1111/j.1540-8191.2008.00641.x

[7] Jensen, H., Jensen, M.O., Smerup, M.H., Vind-Kezunovic, S., Ringgaard, S., Andersen, N.T., Vestergaard, R., Wierup, P., Hasenkam, J.M. and Nielsen, S.L. (2009) Impact of Papillary Muscle Relocation as Adjunct Procedure to Mitral Ring Annuloplasty in Functional Ischemic Mitral Regurgitation. Circulation, 120, S92-S98. http://dx.doi.org/10.1161/CIRCULATIONAHA.108.817833

[8] Watanabe, T., Arai, H., Nagaoka, E., Oi, K., Hachimaru, T., Kuroki, H., Fujiwara, T. and Mizuno, T. (2014) Influence of Procedural Differences on Mitral Valve Configuration after Surgical Repair for Functional Mitral Regurgitation: In Which Direction Should the Papillary Muscle Be Relocated? Journal of Cardiothoracic Surgery, 9, 185. http://dx.doi.org/10.1186/s13019-014-0185-6

[9] Shudo, Y., Matsumiya, G., Sakaguchi, T., Miyagawa, S., Yoshikawa, Y., Yamauchi, T., Takeda, K., Saito, S., Nakatani, S., Taniguchi, K., Izutani, H. and Sawa, Y. (2010) Assessment of Changes in Mitral Valve Configuration with Multidetector Computed Tomography: Impact of Papillary Muscle Imbrication and Ring Annuloplasty. Circulation, 122, S29-S36. http://dx.doi.org/10.1161/CIRCULATIONAHA.109.928002

[10] Kalra, K., Wang, Q., McIver, B.V., Shi, W., Guyton, R.A., Sun, W., Sarin, E.L., Thourani, V.H. and Padala, M. (2014) Temporal Changes in Interpapillary Muscle Dynamics as an Active Indicator of Mitral Valve and Left Ventricular Interaction in Ischemic Mitral Regurgitation. Journal of the American College of Cardiology, 64, 1867-1879. http://dx.doi.org/10.1016/j.jacc.2014.07.988

[11] Sanz, J. and Weinsaft, J.W. (2014) Ischemic Mitral Regurgitation: Is Mitral Valve Physiology Moving from Global to Local? Journal of the American College of Cardiology, 64, 1880-1882. http://dx.doi.org/10.1016/j.jacc.2014.08.029

[12] Jouan, J., Tapia, M., Cook, R.C., Lansac, E. and Acar, C. (2004) Ischemic Mitral Valve Prolapse: Mechanisms and Implications for Valve Repair. European Journal of Cardio-Thoracic Surgery, 26, 1112-1117. http://dx.doi.org/10.1016/j.ejcts.2004.07.049

[13] Nishimura, R.A., Otto, C.M., Bonow, R.O., Carabello, B.A., Erwin 3rd, J.P., Guyton, R.A., O’Gara, P.T., Ruiz, C.E., Skubas, N.J., Sorajja, P., Sundt 3rd, T.M. and Thomas, J.D., American College of Cardiology/American Heart Association Task Force on Practice, G. (2014) 2014 AHA/ACC Guideline for the Management of Patients with Valvular Heart Disease: A Report of the American College of Cardiology/American Heart Association Task Force on Practice Guidelines. Journal of the American College of Cardiology, 63, e57-e185. http://dx.doi.org/10.1016/j.jacc.2014.02.536

[14] Argulian, E. (2015) Valvular Disease, Myocardial Mechanics, and Valve Guidelines. JACC: Cardiovascular Imaging, 8, 382. http://dx.doi.org/10.1016/j.jcmg.2014.12.011

[15] Galli, E., Lancellotti, P., Sengupta, P.P. and Donal, E. (2014) LV Mechanics in Mitral and Aortic Valve Diseases: Value of Functional Assessment beyond Ejection Fraction. JACC: Cardiovascular Imaging, 7, 1151-1166. 
http://dx.doi.org/10.1016/j.jcmg.2014.07.015

[16] Estes Jr., E.H., Dalton, F.M., Entman, M.L., Dixon 2nd, H.B. and Hackel, D.B. (1966) The Anatomy and Blood Supply of the Papillary Muscles of the Left Ventricle. American Heart Journal, 71, 356-362. http://dx.doi.org/10.1016/0002-8703(66)90475-3

[17] Kim, K., Kaji, S., An, Y., Nishino, T., Tani, T., Kitai, T. and Furukawa, Y. (2014) Interpapillary Muscle Distance Independently Affects Severity of Functional Mitral Regurgitation in Patients with Systolic Left Ventricular Dysfunction. The Journal of Thoracic and Cardiovascular Surgery, 148, 434-440.

[18] Nieman, K., Achenbach, S., Pugliese, F., Cosyns, B., Lancellotti, P. and Kitsiou, A. (2015) Cardiac Computed Tomography Core Syllabus of the European Association of Cardiovascular Imaging (EACVI). European Heart JournalCardiovascular Imaging, 16, 351-352. http://dx.doi.org/10.1093/ehjci/jeu298

[19] Zeng, X., Nunes, M.C., Dent, J., Gillam, L., Mathew, J.P., Gammie, J.S., Ascheim, D.D., Moquete, E. and Hung, J. (2014) Asymmetric versus Symmetric Tethering Patterns in Ischemic Mitral Regurgitation: Geometric Differences from Three-Dimensional Transesophageal Echocardiography. Journal of the American Society of Echocardiography, 27, 367-375. http://dx.doi.org/10.1016/j.echo.2014.01.006

[20] Badiwala, M.V., Verma, S. and Rao, V. (2009) Surgical Management of Ischemic Mitral Regurgitation. Circulation, 120, 1287-1293. http://dx.doi.org/10.1161/CIRCULATIONAHA.108.836627 